\title{
Conventional and innovative methods to assess oxidative stress biomarkers in the clinical cardiovascular setting
}

$\overline{\text { Melania Gaggini1 , Laura Sabatino1 \& Cristina Vassalle*,2 }}$

\begin{abstract}
Oxidative stress has a pivotal and widely described role in the onset and progression of atherosclerotic plaque and cardiovascular disease. Many oxidative stress-related biomarkers can be measured in biological samples; however, there are still many aspects that limit the adoption of oxidative stress assessment in clinical laboratory practice. Here, we report an overview of the different sources and main common oxidative stress biomarkers relevant for cardiovascular physiopathology, describing in detail a recently proposed lipidomic assay for ceramide assessment, as a promising future development in oxidative stress evaluation among the many available redox-related tests.
\end{abstract}

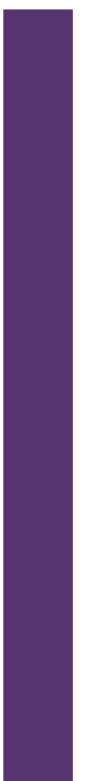

Although atherosclerosis is a multifactorial disorder, oxidative stress is an underlying pivotal physiopathological mechanism common to many cardiovascular risk factors (e.g., hypertension, dyslipidemia, diabetes, obesity, peripheral artery disease, metabolic syndrome and lifestyle determinants such as diet, physical activity and smoking habit) contributing to atherosclerotic damage mainly through its interaction with endothelium, low-density lipoprotein and macrophages [1]. Over the years, several oxidative stress biomarkers have been proposed, mainly reflecting the assessment of oxidation products in biological molecules: lipids, proteins and nucleic acids [2]. Among these, lipid peroxidation biomarkers (e.g., isoprostanes [IsoP] and malondialdehyde [MDA]) are the most used and have attracted great interest, not only for studying physiopathological mechanisms but also for possible implementation in routine clinical laboratory use [2]. Moreover, antioxidant biomarkers can also be evaluated assessing the total antioxidant capacity (TAC); enzymatic antioxidants, such as catalase, glutathione peroxidase, superoxide dismutase; or nonenzymatic markers like vitamins, glutathione and uric acid [2,3]. In view of all the evidence available in experimental studies and clinical trials, the role of oxidative stress in the onset and progression of atherosclerosis and cardiovascular disease is now widely confirmed [4]. Nonetheless, many issues must be further addressed before the introduction of oxidative stress biomarker analysis into clinical laboratory practice, especially with respect to methodology and standardization [5].

The objective of this review is to discuss the most common and potentially relevant oxidative stress biomarkers related to cardiovascular risk and disease. The principles and methods used to assess their levels in biological samples are described, discussing the advantages, disadvantages and promises of available technologies and assays in this field. Other more recent 'omic' technologies - such as genomics, proteomics, metabolomics and lipidomics together with the development of increasingly powerful computational statistical tools, reveal great potential. For this reason, a more detailed description of a recently proposed lipidomic assay for ceramide assessment is reported as an example of a promising future tool in oxidative stress evaluation. Specifically, ceramides, the major molecules of sphingolipid metabolism and lipid second messengers, mediate a cascade of biochemical changes, which leads to increased oxidative stress. These molecules can be measured in plasma and have been associated with chronic degenerative diseases, including cancer and cardiometabolic and neurological conditions.

\section{LIPID PEROXIDATION}

The term 'lipid peroxidation' reflects a process by which oxidants attack cellular membranes, lipoproteins and other molecules that contain lipids. Lipid peroxidation leads to impairment of membrane properties (fluidity and elasticity), cellular damage and cell death. Lipid oxidation produces hydroperoxides, which further originate several reactive intermediates, such as MDA and IsoP. Increased levels of MDA and IsoP in biological fluids have been shown to have important clinical implications because they are associated with many cardiovascular risk factors and coronary artery disease, suggesting that these molecules may represent good biomarkers to assess oxidative stress status in the cardiovascular setting [6].

MDA

MDA is a reactive carbonyl compound and is perhaps the most commonly used biomarker to estimate lipid peroxidation. 
The preanalytical phase is important in oxidative biomarker evaluation, including that of MDA. Particular attention must be given to withdrawal time, blood collection (e.g., temperature) and storage [7]. In fact, ex vivo production may increase MDA levels; the lowest concentrations are found in fresh samples, compared with stored samples [7]. Moreover, the choice of anticoagulant may affect the results, as the lowest MDA values were found in serum collection tubes, followed by heparinized and EDTA plasma tubes [8]. In addition, since hemolysis may increase MDA levels, hemolyzed samples should be avoided [9]. No significant circadian rhythm for circulating MDA and urinary F2-isoprostanes (F2-IsoPs) is evident; therefore, the time of sample collection is not relevant [8].

The reaction of MDA with thiobarbituric acid (TBA) produces thiobarbituric acid reactive substances (TBARS), which give their name to a widespread test. Specifically, the MDA-TBA adduct, formed by the reaction of MDA and TBA under high temperature and acidic conditions in the samples, is measurable by colorimetry or fluorimetry. Based on the use of the TBARS assay in many different clinical and experimental settings, elevated lipid peroxidation has been associated with cardiovascular physiopathology [10-13].

However, the TBARS assay retains low sensitivity and specificity, because it may be affected by many carbonyl group-containing substances, such as sugars, amino acids, bilirubin and albumin, as well as aldehydes other than MDA that can react with TBA to form complexes with similar absorbance or emission wavelengths, which may interfere in colorimetric and fluorimetric MDA assays.

Increased specificity of the TBARS assay may be obtained by separating the MDATBA adduct from TBA adducts of other TBARS substances by high-performance liquid chromatography (HPLC) [14]. MDA blood levels demonstrate a good correlation with 8-iso-prostaglandin F2 $\alpha$ (8-isoPGF $2 \alpha$ ) and 4-hydroxynonenal plasma values [15]. However, the TBARS test results in MDA values higher than those obtained from HPLC techniques, suggesting that spectrophotometric techniques of TBARS may be different than the HPLC approach [16].

Due to the low specificity of TBA reactions, different sequences of pretreatment and derivatization reagents other than TBA have been used for the analysis of MDA by HPLC, chromatography-mass spectrometry (GC-MS), gas chromatography/tandem mass spectrometry (GC$\mathrm{MS} / \mathrm{MS}$ ) and liquid chromatography-tandem mass spectrometry (LC-MS/MS) [6]. Often, these assays do not correlate well with each other. Moreover, even though these methods are more reproducible and reliable than the colorimetric assay, they are also more expensive, time-consuming and labor-intensive; as such, they are still not recommended for processing large sample numbers in a routine laboratory.

\section{IsoP}

F2-isoprostanes are prostaglandin-like substances, the chemically stable end-products of lipid peroxidation formed as a result of free radical-induced peroxidation of arachidonic acid. 8-isoPGF2 $\alpha$, an F2-IsoP with remarkable biological activity (vasoconstrictive and platelet-activating properties), is commonly used as a biomarker for the assessment of oxidative stress status [17]. This molecule can be measured in biological fluids, including plasma and urine, using both MS and ELISAs [18,19]. GC-MS and $\mathrm{LC}-\mathrm{MS} / \mathrm{MS}$ remain the gold standards for measurement of isoP; however, they are expensive and require specialized instrumentation and skilled operators. For these reasons, commercial immunoassays have been developed as cheaper and easier alternatives $[18,19]$. The main advantages of using these commercial ELISA kits are good quality control, assay stability and reproducibility. In ELISA, various antigen-antibody combinations are used, always including an enzyme-labeled antigen or antibody and enzyme activity, which is measured colorimetrically throughout as it modifies the color of the substrate. However, results obtained with ELISA often do not correlate with those obtained by GC-MS $[18,19]$. The choice of antibodies and analytical factors such as the type of sample matrix used for measurement may contribute to the differences observed. It can be hypothesized that GC-MS and ELISAs may not measure the same compounds. In fact, while it is likely that GC-MS analysis measures only a single compound, the antibodies used in ELISA may cross-react with some of the other F2-IsoPs or other potential metabolites.
Nonetheless, this cross-activity might be useful for the estimation of oxidative stress status, because measurement of both the target molecule and its metabolites may be more representative of the overall oxidative stress in vivo.

\section{DIACRON REACTIVE OXYGEN METABOLITES TEST}

The Diacron reactive oxygen metabolites test is a simple commercial colorimetric assay, designed to quantify early peroxidation species (reactive oxygen species) rather than their end products. This test is based on the principle that transition metals have the capacity to catalyze peroxides in the sample under acidic conditions ( $\mathrm{pH} 4.8)$, forming alkoxy and peroxy radicals, which subsequently react with an amine, leading to the formation of colored products that can be measured spectrophotometrically [20].

Reactions require iron ions; thus, samples must be collected as serum and heparinized plasma, but not EDTA plasma or urine. Moreover, the test can be affected by iron-related conditions (iron deficiency or overload or inflammation-related anemia), which may decrease accuracy. The advantages of this assay are its ease of performance, the ability to set it up in all laboratories and the potential for implementation on automated clinical analyzers or microtiter plates, which allows processing of large sample numbers (e.g., for large-scale studies). Also, the stability of the reactive oxygen species in stored serum samples results in reliability; differences between samples that were immediately dosed and aliquots stored for $24 \mathrm{~h}$ at $4^{\circ} \mathrm{C}$ were nonsignificant [7]. Samples give stable results if stored for 2 days at $4^{\circ} \mathrm{C}$ or $-20^{\circ} \mathrm{C}$ [21] and short-term stability (up to 9 days at $-80^{\circ} \mathrm{C}$ ) appears good [21]. Accordingly, we and other authors observed that serum samples showed a very good degree of stability for up to 1 year of storage at -20 and $-80^{\circ} \mathrm{C}[7,22]$. Moreover, the positive correlation of reactive oxygen metabolites with colorectal cancer in liquid nitrogen frozen samples suggested that this biomarker is stable in long-stored samples [23].

\section{ANTIOXIDANTS}

Since oxidative stress is defined as an imbalance between free radical production 
and antioxidant defenses, it is often necessary to assess the counterpart of oxidation, the antioxidant component [20]. This approach provides information on the involvement of the overall oxidative stress status in cardiovascular pathophysiology, but also offers the possibility to monitor the effectiveness of antioxidant interventions. These factors can be measured individually as enzymatic and nonenzymatic antioxidants or the total antioxidant capacity can be evaluated. Activity of antioxidant enzymes including catalase, glutathione peroxidase and superoxide dismutase or nonenzymatic antioxidants like vitamins, glutathione and uric acid, can be estimated in blood. Given the great number of these molecules, quantifying them individually would be excessively time consuming and would require a variety of analytical techniques, instruments and assays. Additionally, interactions between antioxidant dietary compounds, whether in synergy or competition, may be lost with this single approach. For this reason, TAC is often measured. There are many methods for TAC determination, based on different principles. The relative contribution of individual antioxidants to the total antioxidant activity is also variable. Consequently, there might be a considerable difference in the correlation of TAC with other redox biomarkers [24]. The interpretation of results is even more difficult if we take into account that a variety of causes - for instance, disease risk factors, lifestyle characteristics and conditions other than cardiovascular disease, such as lung, cancer and neurodegenerative diseases - may decrease endogenous antioxidant defense, precipitating worsening of redox imbalance. In addition, the capacity of antioxidants against oxidative stress is determined by several properties, including bioavailability, absorption, transport, stability, metabolism and excretion, which may render the interpretation of data quite complex. Moreover, although high levels of antioxidant enzyme activity may be protective against an acutely elevated oxidative stress status, they may also reflect the upregulation of defense mechanisms in response to chronically high levels of oxidants. In particular, increased antioxidant capacity in the samples is not necessarily a meaningful change if it reflects a response to increased oxidative stress, such as is observed in the serum TAC levels of patients with chronic renal failure, which are mainly due to elevated uric acid (a potent antioxidant) in the presence of high MDA and a marked reduction in ascorbate levels $[25,26]$. Moreover, it is important to consider the paradoxical pro-oxidant effects of antioxidants, such as in the case of xanthine oxidase activity for uric acid production, which also may increase oxidative stress $\left(\mathrm{O}_{2}\right.$ and $\mathrm{H}_{2} \mathrm{O}_{2}$ ) as part of the same reaction. Conversely, a decrease in antioxidant capacity may not necessarily be an adverse effect, if it also reflects decreased production of reactive species [20].

\section{COMMON ANTIOXIDANT ASSAYS}

As of now, the most common tests used to assess total antioxidant capacity are the trolox equivalent antioxidant capacity assay (TEAC), the oxygen radical absorbance capacity assay (ORAC) and the ferric ion reducing antioxidant power assay (FRAP).

\section{TEAC}

In indirect methods, colored persistent radicals (used as probes) are reduced by the antioxidant and the color change after the reaction is detected spectrophotometrically. In the TEAC assay, antioxidants in the samples react with 2,2'-azino-bis(3-ethylbenzothiazoline-6-sulfonic acid) (ABTS ${ }^{+*}$ ) radical cation [27]. Limitations of this approach include the fact that the probes are chemically very different from the radicals responsible for the in vivo autoxidation, where the scavenging reaction may not follow the same progression as the one observed in the assay. Moreover, it is not clear if antioxidants in the sample interrupt the chain reaction of autoxidation or if they are simply able to reduce the probe. Therefore, the results obtained with these assays reflect the 'total radical-trapping antioxidant potential' (TRAP, reducing power) rather than the true antioxidant activity. One important advantage, however, is the ductility of the assay, which can be easily adapted with the addition of more revelation time points.

\section{ORAC}

Like other TRAP assays, this test is based on a competitive probe reaction. Specifically, it involves measuring the inhibitory effect of antioxidants, which compete with a fluorescent protein for quenching peroxyl radicals [28]. The oxidation of the fluorescent protein (lag time) and the maximal percentage of this inhibition are then evaluated. Results are quantified by measuring the protection produced by antioxidants. The assay is reliable for the detection of $\alpha$-tocopherol, vitamin $C$, $\beta$-carotene, uric acid, bilirubin and albumin and one of its advantages is its rapidity; however, some antioxidants, such as the lipid-soluble ones, are not well measured by this method.

\section{FRAP}

Indirect methods, based on the reaction of sample antioxidants with inorganic oxidants (e.g., $\mathrm{Fe}^{3+}$ or $\mathrm{Cu}^{2+}$ ), evaluate the reducing capacity, rather than the antioxidant activity. In particular, the FRAP test is based on the reduction of $\mathrm{Fe}^{3+}$ to $\mathrm{Fe}^{2+}$ by the antioxidants in the samples, with the generation of a colored ferrous complex with 2,4,6-tripyridyls-triazine (Fe II-TPTZ), measured spectrophotometrically after a fixed time [29]. Thus, the result depends on the time taken to complete the reaction (4 $\mathrm{min}$ ) and some slower-reacting antioxidants in the sample cannot be fully oxidized. The assay is very reliable for uric acid, $\alpha$-tocopherol, ascorbic acid and bilirubin; however, albumin reacts very slowly and its activity is low. Thus, the assay may lose thiols (e.g., GSH). Moreover, the reaction is nonspecific and nonantioxidant compounds with some redox potential can still induce the reduction of Fe III-TPTZ. The cupric reducing antioxidant capacity test is based on the same principle as the FRAP test but adopts the reduction of $\mathrm{Cu}^{2+}$ ions in the presence of neocuproine and it is able to evaluate thiols, unlike the FRAP method [30].

\section{Other assays}

In addition to these assays, there are many other methods for quantifying the total nonenzymatic plasma antioxidant capacity, such as the hypochlorous-based Diacron OXY-Adsorbent assay, which is based on the capacity of antioxidants in the sample to oppose the oxidant action of added hypochlorous acid [20]. Reaction with an alkyl-substituted aromatic amine produces a pink-colored product that can be detected spectrophotometrically. The concentration of the colored complex is directly propor- 
tional to the concentration of $\mathrm{HClO}$ and indirectly proportional to the antioxidant capacity. The results are expressed as mol of $\mathrm{HClO}$ consumed by $1 \mathrm{ml}$ of sample ( $\mu \mathrm{mol}$ $\mathrm{HClO} / \mathrm{ml}$ ). Values lower than $350 \mu \mathrm{mol}$ $\mathrm{HClO} / \mathrm{ml}$ suggest a reduced antioxidant capacity. This test is especially suitable for the measurement of low molecular weight compounds (e.g., uric acid), vitamins $\mathrm{C}$ and E and proteins (e.g., albumin). We observed that there was a weak but significant linear correlation between the OXY-Adsorbent test and FRAP ( $r=0.22 ; p<0.05)$ [31]; however, another study evidenced that the OXY-Adsorbent test did not correlate with other antioxidant methods [32]. Nonetheless, this fact can represent an additional utility, as the test can be complementary to other antioxidant assays in the assessment of sample overall antioxidant capacity.

\section{AN EXAMPLE OF PROMISING OXIDATIVE-RELATED LIPIDOMICS: THE PLASMA CERAMIDE ASSAY}

Ceramides are the basic structural units of all sphingolipids and are a class of bioactive lipids that regulate several cell functions. Ceramide species are produced by six fatty acyl selective ceramide synthases, starting from fatty acids and serine or sphingomyelin (Figure 1A).

Ceramides are secondary messengers that regulate cellular differentiation, apoptosis and proliferation and also activate a variety of signaling cascades (Figure 1B) [33]. Emerging reports indicate that oxidative stress and ceramides are correlated [34]. In particular, ceramides increase oxidant production, acting directly on mitochondria by the inhibition of isolated mitochondrial electron transport at complex III. This inhibition leads to an increase in production of reactive oxygen species (Figure 1B) [35]. Circulating ceramides could be used as markers of several inflammatory and cardiovascular diseases; their concentration in serum strongly correlates with future adverse cardiovascular events such as acute myocardial infarction and stroke [36].

In the circulation, ceramides are transported by lipoproteins and are implicated in platelet activation and endothelial dysfunction via the uncoupling of nitric oxide signaling pathways [37,38]. Untargeted

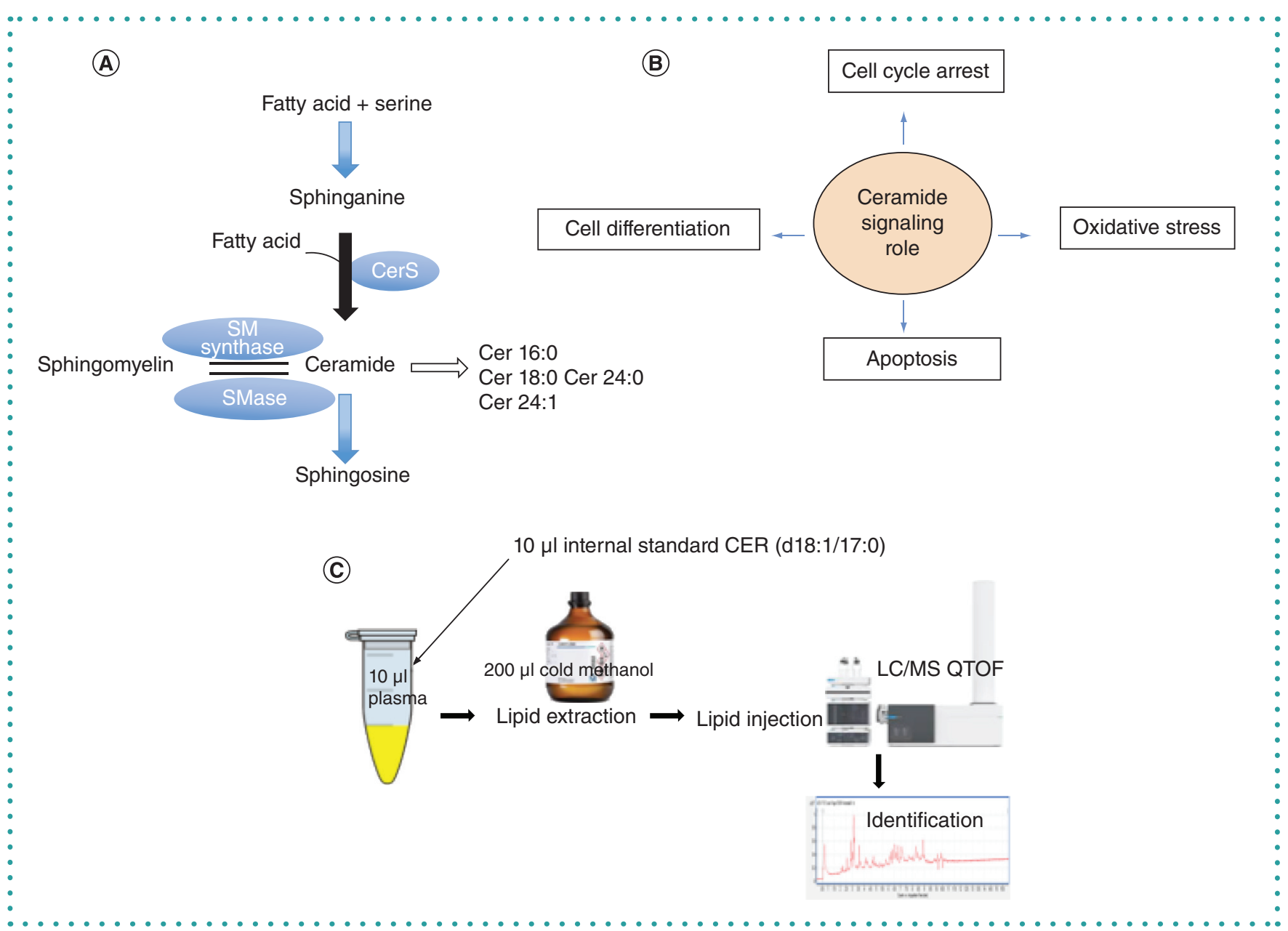

Figure 1. Ceramide synthesis, main biological roles and evaluation by high-performance liquid chromatography assay. (A) Schematic representation of ceramide synthesis. Serine and fatty acyl are converted into sphinganine, which is, in turn, converted to dihydroceramide by CerS, and finally reduced to ceramide by dihydroceramide desaturase. Ceramide is also produced by SMase through SM degradation in the SMase pathway. SMases converts ceramide in sphingosine. There are six different mammalian ceramides (CerS1-6), each of which produces a subset of ceramides that differ in their fatty acyl chain length. (B) Signaling role of ceramides. Cellular Cer levels induce cell cycle arrest, cell differentiation, apoptosis and oxidative stress. (C) Detailed procedure for high-performance liquid chromatography evaluation of ceramides in plasma. CerS: Ceramide synthase; SM: Sphingomyelin; SMase: Sphingomyelinase. 


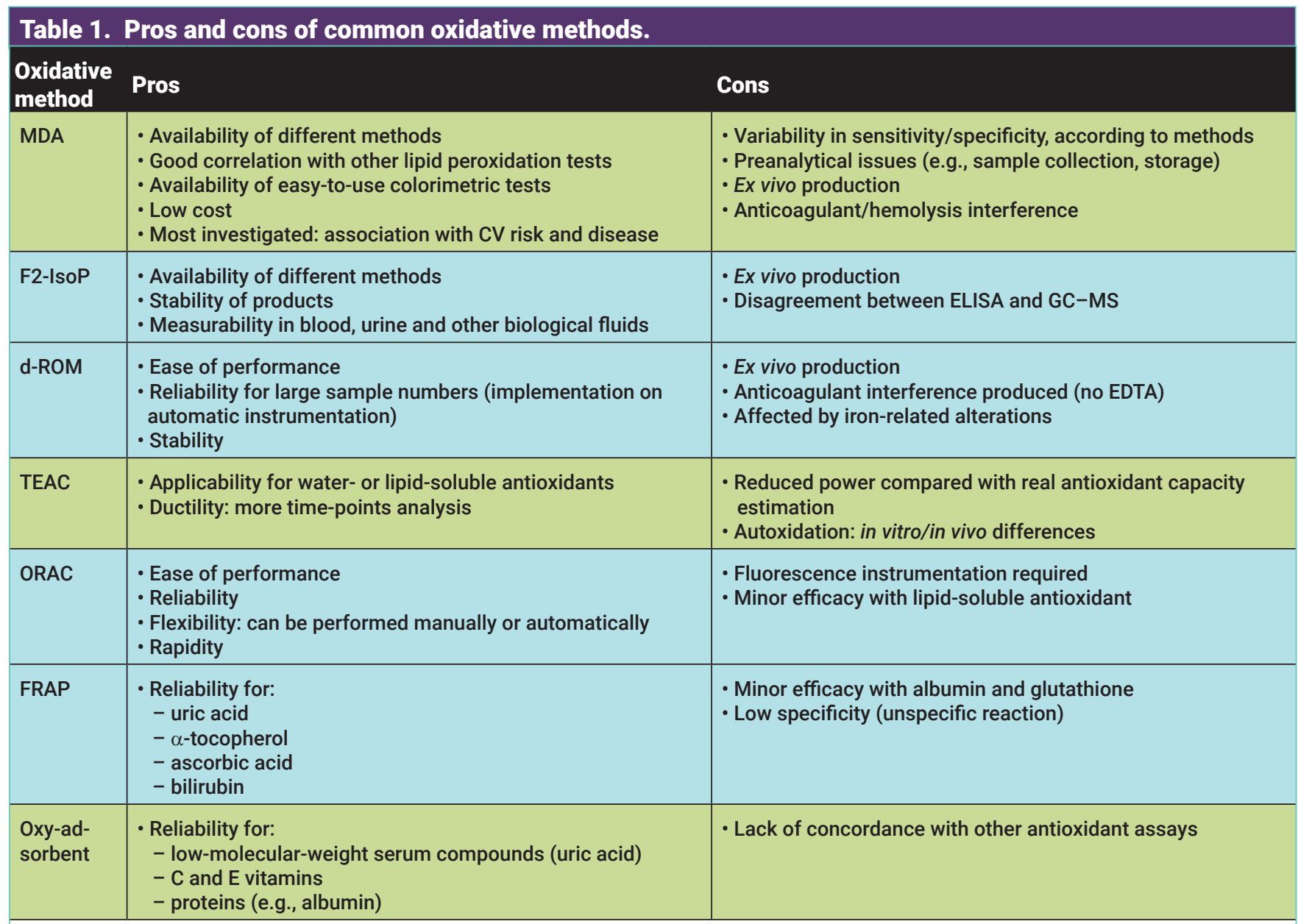

CV: Cardiovascular; d-ROM: Diacron reactive oxygen metabolite; F2-IsoP: F2-isoprostane; FRAP: Ferric ion reducing antioxidant power assay; MDA: Malondialdehyde; ORAC: Oxygen radical absorbance capacity assay; TEAC: Trolox equivalent antioxidant capacity assay.

metabolomic analysis was helpful to develop a ceramide risk score, which combined the plasma concentrations of N-palmitoylsphingosine (Cer(16:0)), N-stearoyl-sphingosine (Cer(18:0)), N-nervonoyl-sphingosine (Cer(24:1)) and $\mathrm{N}$-lignoceroyl-sphingosine (Cer(24:0)); this method proved reliable to assess cardiovascular risk in different populations [39-42]. This procedure is described in detail in Figure 1C.

The measurement of ceramides could be done utilizing LC-MS and LC-MS/MS, LC-ESI-MS/MS, gas-liquid chromatography or fast liquid chromatography quadrupole time-of-flight mass spectrometry. Clearly these techniques are not widespread, are still expensive and require specialized instrumentation and operators.

Limitations of measuring ceramide dosage are related to the extraction phase of the sample, which depends on the operator. Moreover, a possible ionic suppression caused by the nature of the matrix must be considered. There are no reference values for ceramides, which should be evaluated in different physiopathological settings. Data on within- and between-person biological variability are still lacking, as well as changes according to age and gender.

\section{PEARLS \& PITFALLS}

Different assays in the oxidative stress field are available and include:

- Direct detection of reactive oxygen species (infrequently used due to difficulty stemming from the short half-life and high reactivity of radical species);

- Indirect oxidative stress determination (measures stable products of oxidative damage to biomolecules; in each clinical and experimental context, the selection of a suitable marker of oxidative stress is critical, because proteins, lipids or DNA may reflect different molecular and cellular injures);
- Antioxidant status (enzymatic antioxidant activities, nonenzymatic antioxidant levels; if singularly evaluated, synergy or competition between compounds may be unappreciated; contribution of each antioxidant to the total antioxidant capacity may vary).

Moreover, ongoing studies in the field of genomics, proteomics, metabolomics and lipidomics, together with advances in bioinformatics and artificial intelligence, have exceptional potential to define further critical context-specific transcripts, proteins and metabolites. Nonetheless, many aspects must be improved before the introduction of oxidative stress status assessment into clinical laboratory practice.

The evaluation of many blood oxidative stress assays identifies several advantages, including easy collection and assay execution, relatively low cost and the facility to test many samples in laboratory routine or large studies. However, sample 
collection, processing and storage should be performed with extreme attention and must be standardized to ensure stability and to avoid the possibility of biomolecules becoming oxidized ex vivo (Table 1).

Regarding method agreement, despite mass spectrometry providing higher sensitivity, specificity and becoming more accessible, it is expensive in terms of equipment and staff training; therefore, antibody-based assays, which often retain lower specificity, remain more widely used. In general, there is a lack of standardization for most of the proposed methods. For this reason, it is important to start large-scale multicenter laboratory studies to standardize the methodological and analytical issues, develop and disseminate good laboratory practices, produce large data sets, facilitate the establishment of reference values for each biomarker and validate their reliability in translational medicine applications. In this context, some large-scale multilaboratory validation studies have been conducted, such as the European Comet Assay Validation Group (ECVAG; $\gamma$-ray irradiated calibration samples and three coded samples of human cells, containing cells with different amounts of 8-oxoguanine in their DNA), European Standards Committee on Oxidative DNA Damage (ESCODD; samples of 8-oxodGcontaining oligonucleotides with different ratios of 8-oxodG/2'-deoxyadenosine), the Biomarkers of Oxidative Stress Study (BOSS; effects of $\mathrm{CCl} 4$ on concentrations of TBARS, MDA, IsoP, protein carbonyls, methionine sulfoxidation, tyrosine products, 8-hydroxy-2'-deoxyguanosine, leukocyte DNA-MDA adducts and DNA-strand breaks, using acute $\mathrm{CCl} 4$ poisoning in a rodent model) and the COST Action B35 'Lipid Peroxidation Associated Disorders' study (human plasma samples exposed to UVA irradiation at different doses, analyzed for MDA, 4-hydroxynonenal and IsoP). All these groups aimed to assess inter-method and inter-laboratory variations in a prospective study of measurement standardization, assessing precision, accuracy and result comparison between laboratories [43-46]. However, an important consideration is that these ex vivo experimental results would need to be reproduced with the analysis of oxidative stress-induced damage in vivo. In this context, another study faced validation issues of PON1 enzyme activity assays for longitudinal studies in serum or heparinized plasma (assay temperature, storage up to 7 years at $-80^{\circ} \mathrm{C}$, freeze-thaw cycles, type of specimen, and assay variability were evaluated for 4 PON1 substrate-specific assays using samples from two pediatric cohorts and laboratory volunteers), but included only two laboratories [47].

In evaluating the overall oxidative stress status, it is important to consider that 'oxidative stress' is a unifying mechanism underlying all the cardiovascular risk factors, (e.g., smoking habit, hypertension, Type 2 diabetes, dyslipidemia). Thus, all the oxidative stress-related biomarkers may be questionable due to their low specificity; however, this characteristic may paradoxically represent an additional value, which arises from its representing underlying indicators of several conditions, rather than a specific one, thus revealing the potential of integrating all risk factors into a global cardiovascular risk assessment with prognostic relevance. While there is a wide spectrum of oxidative stress biomarkers, it is clear that the different biomarkers available do not measure identical aspects of oxidative stress. Consequently, the overall oxidative stress status cannot be defined by any unique universal biomarker, because a single parameter can only partially describe the true picture of multilevel oxidative stress. However, this biochemical heterogeneity might be advantageous to catch the overall effects of oxidative stress in a multimarker approach (Figure 2). It is generally recommended that the antioxidant counterpart is evaluated beside oxidant parameters. In this context, integrative comprehensive indices, using more than one criterion and including both pro- and antioxidant biomarkers, may give a more correct and powerful evaluation of overall oxidative stress, with higher sensitivity to physiopathological modifications. For example: the OSI index measures the percentage ratio of total peroxide level to total antioxidant capacity (measured by using FRAP) [48]; the OXY-SCORE is calculated by subtracting the protective defenses (GSH, $\alpha$ - and $\gamma$-tocopherol levels, and antioxidant capacity) from the oxidative damage score (plasma free and total malondialdehyde, GSSG/GSH ratio, and urine F2-IsoPs) $[49,50]$. Similarly, the oxidative-INDEX is obtained by subtracting the total antioxidant capacity (measured with the commercial Diacron
OXY-Adsorbent test) standardized variable from the reactive oxygen metabolites (measured with the commercial Diacron reactive oxygen metabolites) standardized variable [20]. The use of different biomarkers for the assessment of a global oxidative stress score does not always give the same results. In the creation of a global index, it is difficult to advise which specific biomarkers must be considered, accounting for the particular setting in which they are evaluated in order to avoid possible misleading interpretation. As an example, in the chronic venous insufficiency context, different oxidative stress biomarkers related to antioxidant defenses (total thiols, reduced glutathione, uric acid, total antioxidant capacity, catalase), oxidative injury (MDA-bound protein, protein carbonyls, advanced oxidation products and 3-nitrotyrosine) and enzyme activity (xanthine oxidase and myeloperoxidase) were evaluated to define an overall index of oxidative status. Normalized and standardized parameters, which showed a significant statistical difference between chronic venous insufficiency patients and controls (thiols, MDA-bound proteins, protein carbonyls and catalase activity) were proposed for the calculation of a global index in this specific clinical condition [51]. In another case, a multiple factor analysis statistical approach was utilized, allowing simultaneous study of many parameters (including but not limited to those related to oxidative stress and inflammation) to evaluate biomarker changes after an Ironman race [52]. This integrative approach revealed that changes in the oxidative index status were associated with variations in IL-8 and in the cardiac marker $\mathrm{N}$-terminal pro-B-type natriuretic peptide (NT-proBNP), related to the physiological response following strenuous endurance exercise in athletes. In particular, subjects who showed a higher improvement of the oxidative index after the race exhibited small changes in NT-proBNP and IL-8 values, whereas subjects with minimal variation in the oxidative index had a marked post-race increase in NT-proBNP and IL-8 concentrations. The strategy to evaluate larger biomarker panels (not limited to those relating to the redox balance) appears reasonable and may help to select more relevant and significant biomarkers in specific clinical settings (Figure 2). 


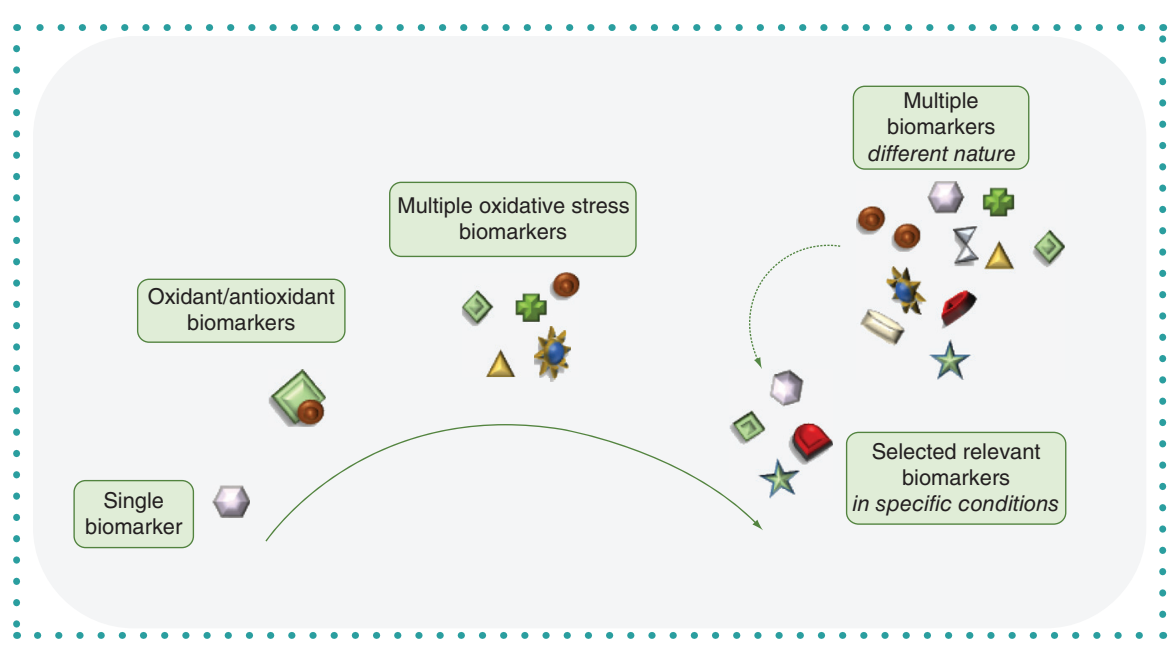

It will also be important to evaluate the additive value of the oxidative stress assessment over the traditional risk scores used. An attempt in this direction is the evaluation of urinary 8-hydroxy-2'deoxyguanosine and 8-isoprostane when added to the 10-year cardiovascular disease mortality risk ESC-SCORE (developed for 10-year cardiovascular disease mortality risk prediction and including age, sex, systolic blood pressure, total cholesterol/ high-density lipoprotein-cholesterol ratio and current smoking status) in the ESTHER cohort [53]. Compared to the reference model ESC-SCORE, the model ESC-SCORE+8isoprostane resulted in a Net Reclassification Index of $5.3 \%$. Moreover, further addition of oxidized guanine/guanosine levels to the ESC-SCORE+8-isoprostane resulted in an Net Reclassification Index corresponding to $5.5 \%$ when compared with the reference model ESC-SCORE+8-isoprostane.

As levels of oxidative stress biomarkers can be affected by commonly used therapy and interventions, directions for future research should also include the potential to tailor individual and personalized management and allow patient stratification based on pathogenic mechanisms rather than disease severity. Negative results obtained by interventional studies with antioxidants in primary and secondary prevention of cardiovascular disease may relate to an incorrect identification of candidates for the antioxidant supplementation, as a basal determination of blood oxidative stress status is seldom performed. As patients with higher oxidative stress status would benefit more from antioxidant supplementation, a baseline evaluation in this sense could be considered while designing future studies.

\section{FUTURE PERSPECTIVE}

Despite all the available oxidative stressrelated biomarkers and despite their confirmed predictive value in cardiovascular disease, almost none have entered routine clinical practice. Before transfer of oxidative stress assays into clinical laboratory practice, there are several issues that require further focus, which can be the subject of future research and validation in the next few years:

- Standardization of pre-analytical issues, which may greatly affect measurements;

- Agreement on which methods to use;

- Assay standardization;

- Choice of biomarkers (single or multimarker panels) to be used in specific experimental/clinical conditions;

- Evaluation of additional value of oxidative stress biomarkers with respect to established models of cardiovascular risk prediction;

- Possible use of oxidative stress biomarkers as targets of therapy and interventions.

\section{CONCLUSION}

There is robust and unequivocal evidence validating the role of oxidative stress in cardiovascular physiopathology, although many limitations hamper the immediate transfer of these tools into routine clinical laboratory testing. Importantly, a reasonable strategy would be to identify a biomarker panel (including, but not limited to, oxidative stress-related parameters) that better reflects the key physiopathological
Figure 2. Different strategies to assess in vivo oxidative stress. The availability of different biomarkers of oxidative stress may represent a feasible opportunity for their implementation in clinical practice, although validation issues remain to be resolved. The wide diversity in oxidative stress biomarkers does not mean that one is better than others, but must be taken into account when choosing which biomarker to use in specific conditions. Evaluation of larger biomarker panels will help to give a more comprehensive scenario and to select those biomarkers that are more relevant in a specific clinical setting, revealing the incremental value of oxidative stress biomarkers, in addition to conventional models of cardiovascular risk prediction and power, as targets for a new generation of redox therapies.

processes pertinent to each specific clinical setting.

Thus, if it remains premature to assign a clinical validity for each of the reported oxidative stress biomarkers, this should not be discouraging but rather indicates a wide scope for improvement and discovery to render oxidative biomarkers suitable for routine clinical practice in the near future.

\section{AUTHOR CONTRIBUTIONS}

C Vassalle conceived and designed the manuscript and made a substantial contribution in drafting the article and the revised version. All the authors contributed intellectual content to the manuscript and gave approval to the final version.

\section{FINANCIAL \& COMPETING INTERESTS DISCLOSURE}

The authors have no relevant affiliations or financial involvement with any organization or entity with a financial interest in or financial conflict with the subject matter or materials discussed in the manuscript. This includes employment, consultancies, honoraria, stock ownership or options, expert testimony, grants or patents received or pending, or royalties.

No writing assistance was utilized in the production of this manuscript.

\section{OPEN ACCESS}

This work is licensed under the AttributionNonCommercial-NoDerivatives 4.0 Unported License. To view a copy of this license, visit http://creativecommons.org/licenses/ by-nc-nd/4.0/ 


\section{EXECUTIVE SUMMARY}

- Oxidative stress has a pivotal role in the onset and progression of atherosclerotic plaque and cardiovascular disease.

- Many oxidative stress-related biomarkers can be measured in biological samples, although several aspects need to be addressed before implementation of oxidative stress measurements in clinical laboratory practice.

- Issues related to advantages, disadvantages and promises of available technologies and assays in the oxidative stress field are discussed.
- A recently proposed lipidomic assay for ceramide assessment is reported in detail among the many available redoxrelated tests, as an example of promising future development in oxidative stress evaluation.

\section{REFERENCES}

Papers of special note have been highlighted as: • of interest; $\cdot$ - of considerable interest

1. Siti HN, Kamisah Y, Kamsiah J. The role of oxidative stress, antioxidants and vascular inflammation in cardiovascular disease (a review). Vascul. Pharmacol. 71, 40-56 (2015).

.. Overview of the relationship between oxidative stress, inflammation, endothelial dysfunction, cardiovascula risk factors and atherosclerosis.

2. Frijhoff J, Winyard PG, Zarkovic $\mathrm{N}$ et al. Clinical relevance of biomarkers of oxidative stress. Antioxid. Redox Signal. 23(14), 1144-1170 (2015).

3. Mastorci F, Traghella I, Sabatino L, Pingitore A, Ndreu $\mathrm{R}$, Vassalle C. Oxidative stress and cardiovascular risk and prevention in children and adolescents. Chapter 1. In: Oxidative Stress in Heart Diseases. Chakraborti S (Ed.). Springer, Singapore (2019).

4. Cervantes Gracia K, Llanas-Cornejo D, Husi H. CVD and oxidative stress. J. Clin. Med. 6(2), 22 (2017).

5. Vassalle C. Oxidative stress and cardiovascular risk prediction: the long way towards a "radical" perspective. Int. J. Cardiol. 273, 252-253 (2018).

6. Ito $\mathrm{F}$, Sono $\mathrm{Y}$, Ito $\mathrm{T}$. Measurement and clinical significance of lipid peroxidation as a biomarker of oxidative stress: oxidative stress in diabetes, atherosclerosis, and chronic inflammation. Antioxidants (Basel) 8(3), 72 (2019).

7. Vassalle C, Boni C, Di Cecco P, Ndreu R, Zucchelli GC. Automation and validation of a fast method for the assessment of in vivo oxidative stress levels. Clin. Chem. Lab. Med. 44(11), 1372-1375 (2006)

8. Tsikas D, Rothmann S, Schneider JY et al. Development, validation and biomedical applications of stable-isotope dilution GC-MS and GC-MS/MS techniques for circulating malondialdehyde (MDA) after pentafluorobenzyl bromide derivatization: MDA as a biomarker of oxidative stress and its relation to 15[S]-8-iso-prostaglandin F2 and nitric oxide [NO] J. Chromatogr. B Analyt. Technol. Biomed. Life Sci. 1019, 95-111 (2016).

9. Dreissigacker U, Suchy MT, Maassen N, Tsikas D. Human plasma concentrations of malondialdehyde [MDA] and the F2-isoprostane 15[S]-8-iso-PGF[2alpha] may be markedly compromised by hemolysis: evidence by GC-MS/MS and potential analytical and biological ramifications. Clin. Biochem. 43(1-2), 159-167 (2010).

10. Vassalle C, Lubrano V, L'Abbate A, Clerico A. Determination of nitrite plus nitrate and malondialdehyde in human plasma: analytical performance and the effect of smoking and exercise. Clin. Chem. Lab. Med. 40(8), 802-809 (2002)

11. Freitas DA, Rocha-Vieira E, Soares BA et al. High intensity interval training modulates hippocampa oxidative stress, BDNF and inflammatory mediators in rats. Physiol. Behav. 184, 6-11 (2018)

12. Craig A, Mels $C M C$, Kruger R. Thiobarbituric acid-reactive substances relate to arterial stiffness and blood pressure in 6 - to 8 -year-old boys stratified by materna risk. Free Radic. Res. 52(2), 180-187 (2018).

13. Taty Zau JF, Costa Zeferino R, Sandrine Mota $\mathbf{N}$ et al. Exercise through a cardiac rehabilitation program attenuates oxidative stress in patients submitted to coronary artery bypass grafting. Redox Rep. 23(1), 94-99 (2018)

14. Tsikas D. Assessment of lipid peroxidation by measuring malondialdehyde (MDA) and relatives in biological samples: analytical and biological challenges. Anal. Biochem. 524, 13-30 (2017).

15. Tsikas D, Suchy MT, Niemann J et al. Glutathione promotes prostaglandin $\mathrm{H}$ synthase (cyclooxygen- ase)-dependent formation of malondialdehyde and 15[S]-8-iso-prostaglandin F2 $\alpha$. FEBS Lett. 586(20), 3723-3730 (2012).

16. Moselhy HF, Reid RG, Yousef S, Boyle SP. A specific accurate, and sensitive measure of total plasma accurate, and sensitive measure of total plasma
malondialdehyde by HPLC. J. Lipid Res. 54(3), 852-858 (2013).

17. Czerska M, Zieliński M, Gromadzińska J. Isoprostanes - a novel major group of oxidative stress markers. Int J. Occup. Med. Environ. Health 29(2), 179-190 (2016).

18. Klawitter J, Haschke M, Shokati T, Klawitter J, Christians U. Quantification of 15-F2t-isoprostane in human plasma and urine: results from enzyme-linked immunoassay and liquid chromatography/tandem mass spectrometry cannot be compared. Rapid Commun Mass Spectrom. 25(4), 463-468 (2011).

19. Smith KA, Shephard J, Wakil A, Kilpatrick ES. A comparison of methods for the measurement of 8-isoPGF[2 $\alpha$ ]: a marker of oxidative stress. Ann. Clin. Biochem. 48(2), 147-154 (2011).

20. Vassalle $C$. An easy and reliable automated method to estimate oxidative stress in the clinical setting Methods Mol. Biol. 477, 31-39 (2008).
Mo estimate oxidative stress in the clinical

21. Jansen EHJM, Beekhof PK, Cremers JWJM, Viezeliene D, Muzakova V, Skalicky J. Short-term stability of biomarkers of oxidative stress and antioxidant status in human serum. ISRN Biomark. 2013, 1-5 (2013).

22. Jansen EHJM, Beekhof PK, Viezeliene D, Muzakova V Skalicky J. Long term stability of cancer biomarkers of oxidative stress, redox status, homocysteine, CRP and liver enzymes in human serum. Biomark. Med. 9(5), 425-432 (2015).

23. Leufkens AM, van Duijnhoven FJB, Woudt SHS et al Biomarkers of oxidative stress and risk of developing colorectal cancer: a cohort-nested case-control study in the EPIC study. Am. J. Epidemiol. 175(7), 653-663 (2012).

24. Lee SG, Wang T, Vance TM et al. Validation of analytical methods for plasma total antioxidant capacity by com-
paring with urinary 8-isoprostane level. J. Microbiol. paring with urinary 8-isoprostane level
Biotechnol. 27(2), 388-394 (2017).

Comparison of four conventional analytical methods for measuring total antioxidant capacity (ABTS, DPPH FRAP and ORAC assays) by comparing with urinary 8-isoprostane concentration.

25. Jackson P, Loughrey CM, Lightbody JH, McNamee PT, Young IS. Effect of hemodialysis on total antioxidant capacity and serum antioxidants in patients with chronic renal failure. Clin. Chem. 41(8), 1135-1138 (1995).

26. Vassalle C, Mazzone A, Sabatino L, Carpeggiani C. Uric acid for cardiovascular risk: Dr. Jekyll or Mr. Hide? Diseases 4(1), 12 (2016).

27. Re R, Pellegrini N, Proteggente A, Pannala A, Yang $M$, Rice-Evans $C$. Antioxidant activity applying an im$M$, Rice-Evans C. Antioxidant activity applying an im-
proved ABTS radical cation decolorization assay. Free proved ABTS radical cation decolorization assay.
Radic. Biol. Med. 26(9-10), 1231-1237 (1999).

28. Cao G, Alessio HM, Cutler RG. Oxygen-radical absorb ance capacity assay for antioxidants. Free Radic. Biol. Med. 14(3), 303-311 (1993).

29. Benzie IFF, Strain JJ. The ferric reducing ability of plasma (FRAP) as a measure of 'antioxidant power':

30. Apak R, Güclü K, Ozyürek M, Karademir SE. Novel total antioxidant capacity index for dietary polyphenols and vitamins $C$ and $E$, using their cupric ion reducing capability in the presence of neocuproine: CUPRAC method. J. Agric. Food Chem. 52(26), 7970-7981 (2004).

31. Vassalle C, Masini S, Carpeggiani C, L'Abbate A, Boni C, Zucchelli GC. In vivo total antioxidant capacity: comparison of two different analytical methods. Clin. Chem. Lab. Med. 42(1), 84-89 (2004).
32. Jansen EH, Ruskovska T. Comparative analysis of serum (anti)oxidative status parameters in healthy persons. Int. J. Mol. Sci. 14(3), 6106-6115 (2013).

33. Riboni L, Viani P, Bassi R, Prinetti A, Tettamanti G. The role of sphingolipids in the process of signal transduction. Prog. Lipid Res. 36(2-3), 153-195 (1997).

34. Andrieu-Abadie N, Gouazé V, Salvayre R, Levade T. Ceramide in apoptosis signaling: relationship with oxidative stress. Free Radic. Biol. Med. 31(6), 717-728 (2001).

35. Summers SA. Ceramides in insulin resistance and lipotoxicity. Prog. Lipid Res. 45(1), 42-72 (2006).

36. Meeusen JW, Donato LJ, Bryant SC, Baudhuin LM, Berger PB, Jaffe AS. A novel predictor of major adverse cardiovascular events after coronary angiography. Arterioscler. Thromb. Vasc. Biol. 38(8), 1933-1939 (2018)

37. Napp M, Zendzian-Piotrowska M, Błachnio-Zabielska A, Zabielski P, Kurek K, Górski J. Myocardial infarction differentially alters sphingolipid levels in plasma, erythrocytes and platelets of the rat. Basic Res. Cardiol. 107(6), 294 (2012).

38. Mugabo Y, Mukaneza Y, Renier G. Palmitate induces C-reactive protein expression in human aortic endothelial cells. Relevance to fatty acid-induced endothelial dysfunction. Metabolism 60(5), 640-648 (2011).

39. Arasov K, Ekroos K, Suoniemi M et al. Molecular lipids identify cardiovascular risk and are efficiently lowered by simvastatin and PCSK9 deficiency. J. Clin Endocrinol. Metab. 99(1), e45-e52 (2014).

40. Wang DE, Toledo E, Hruby A et al. Plasma ceramides, Mediterranean diet, and incident cardiovascular disease in the PREDIMED trial (Prevención cor Mediterránea). Circulation 135(21), 2028-2040 (2017).

41. Havulinna AS, Sysi-Aho M, Hilvo $M$ et al. Circulating ceramides predict cardiovascular outcomes in the population-based FINRISK 2002 cohort. Arterioscler. Thromb. Vasc. Biol. 36(12), 2424-2430 (2016). Association between ceramides and major adverse cardiovascular events in a general population.

42. Laaksonen R, Ekroos K, Sysi-Aho M et al. Plasma ceramides predict cardiovascular death in patients with stable coronary artery disease and acute coronary syndromes beyond LDL-cholesterol. Eur. Heart J. 37(25) 1967-1976 (2016)

43. Johansson C, Møller P, Forchhammer L. An ECVAG tria on assessment of oxidative damage to DNA measured by the comet assay. Mutagenesis 25(2), 125-132 (2010).

44. Riis B. Comparison of results from different laboratories in measuring 8-oxo-2'-deoxyguanosine in synthetic oligonucleotides. Free Radic. Res. 36(6), 649-659 (2002).

45. Breusing N, Grune T, Andrisic L et al. Biomarkers of ox idative stress study II: are oxidation products of lipids, proteins, and DNA markers of $\mathrm{CCl} 4$ poisoning? Free Radic. Biol. Med. 38(6), 698-710 (2005).

46. Breusing N, Grune T, Andrisic L et al. An inter-laboratory validation of methods of lipid peroxidation measurement in UVA-treated human plasma samples. Free Radic. Res. 44(10), 1203-1215 (2010).

47. Huen K, Richter R, Furlong C, Eskenazi B, Holland N. Validation of PON1 enzyme activity assays for longitudinal studies. Clin. Chim. Acta 402(1-2), 67-74 (2009).

48. Yanik M, Erel O, Kati M. The relationship between potency of oxidative stress and severity of depression. Acta Neuropsychiatr. 16, 200-203 (2004).

49. Veglia F, Cighetti G, De Franceschi M et al. Age- and gender-related oxidative status determined in healthy subjects by means of OXY-SCORE, a potential new comprehensive index. Biomarkers 11(6), 562-573 (2006). 
50. Veglia F, Cavalca V, Tremoli E. OXY-SCORE: a global index to improve evaluation of oxidative stress by index to improve evaluation of oxidative stress by Biol. 594, 197-213 (2010).

51. Condezo-Hoyos $\mathrm{L}$, Rubio $\mathrm{M}$, Arribas $\mathrm{SM}$ et al. A plasma oxidative stress global index in early stages of chronic venous insufficiency. J. Vasc. Surg. 57(1), 205-213 (2013).

.. Evaluation of an oxidative stress multipanel to identify more significant parameters for the calculation of a global index in chronic venous insufficiency.
52. Vassalle $\mathrm{C}$, Piaggi P, Weltman $\mathrm{N}$ et al. Innovative approach to interpret the variability of biomarkers afte ultra-endurance exercise: the multifactorial analysis. Biomark. Med. 8(6), 881-891 (2014).

- Multipanel assessment to identify most significan biomarkers (including, but not limited to, those related biomarkers (including, but not limited to, those related to oxidative stress

53. Xuan Y, Gào X, Holleczek B, Brenner H, Schöttker $B$. Prediction of myocardial infarction, stroke and cardiovascular mortality with urinary biomarkers of oxidative stress: results from a large cohort study. Int. J. Cardiol. 273, 223-229 (2018).

.. Identification of oxidative stress biomarkers to improve cardiovascular disease outcome prediction. 